\title{
Web Accessibility Interventions for Citizens Living with Cognitive Impairment: A Rapid Evidence Assessment
}

Paul Flynn ( $\sim$ paul.flynn@nuigalway.ie)

National University of Ireland Galway https://orcid.org/0000-0002-0957-8605

Sara Gartland

National University of Ireland Galway

Joe Cullen

Arcola Research

Maria Ana Carneiro

Universidade Católica Portuguesa: Universidade Catolica Portuguesa

Jose de Sousa Fialho

Universidade Católica Portuguesa: Universidade Catolica Portuguesa

Greg Holloway

Arcola Research

Clare Cullen

Arcola Research

Emma Hamilton

Arcola Research

Amy Harris

Arcola Research

\section{Protocol}

Keywords: Web accessibility, cognitive impairment

Posted Date: November 19th, 2021

DOl: https://doi.org/10.21203/rs.3.rs-994747/v1

License: (c) (i) This work is licensed under a Creative Commons Attribution 4.0 International License.

Read Full License 


\section{Abstract}

\section{Background}

There is a clear and pressing need to understand the barriers to technology user experience, particularly in relation to people with cognitive disabilities. The COVID-19 pandemic and subsequent expansion of digital technologies associated with education, wellness and employment only makes the need to understand how people with cognitive disabilities interact with such technologies, within the naturalistic context of their lifeworld, increasingly urgent. Therefore a necessary first step is to develop a baseline understanding of the current state of web accessibility for people with cognitive disabilities. Thus, the purpose of this review is to conduct baseline research to understand the factors and processes that inhibit access to online content and services for people with cognitive impairments.

\section{Methods}

This systematic, rapid evidence assessment, review will employ a search strategy using defined terms within agreed search strings in the following databases: Web of Science, SCOPUS, EBSCOhost, ERIC and ProQuest. Internet searching through Google Scholar will be carried out as well as forward and backward tracking of citations from studies that are included in the review. All results, screening process results will be documented in tabular form and communicated in a PRISMA flow diagram. In addition, the research team consider it necessary to carry out a grey literature search due to the nature of the work being investigated. The expertise within the research team indicated that many programmes that support people with cognitive impairments do not formally report their work through academic dissemination pathways. Consequently it is intended that a grey literature search will be carried out to supplement that findings of study. In contrast to the focus on studies published in English for peer review returns, the grey literature search will actively seek out returns across all languages of the European Union.

\section{Discussion}

This rapid review protocol will focus on citizens ages 9 and up who live with cognitive impairment and establish a baseline for best practice in supporting web accessibility for people with cognitive impairments. It will achieve this by providing a time limited state-of-the-art evidence report, specific to the challenges people with cognitive impairment, that will help those involved in policy development, policy response initiatives and localised activity. It is intended that, depending on the outcome of the review process, additional opportunities for innovation and/or research may be communicated to relevant stakeholders and policy makers.

\section{Systematic Review Registration}

This protocol has been submitted to the International Prospective Register for Systematic Reviews PROSPERO, and is currently under review. Reference No. 269245. 


\section{Background}

The COVID-19 pandemic sharply increased the embedded nature of digital technologies in daily life and simultaneously amplified existing social, educational, and economic inequities (WEF, 2020). Prior to the pandemic, research highlighted the importance of better understanding the barriers to technology user experience, particularly in relation to people with cognitive disabilities (Barnard \& Beyer, 2009; Collings, et.al., 2018; Neven, 2010), a group that is often marginalised in society and in research (Borg, et.al., 2015). The COVID-19 pandemic and subsequent expansion of digital technologies associated with education, wellness and employment only makes the need to understand how people with cognitive disabilities interact with such technologies, within the naturalistic context (Flynn et al., 2016) of their lifeworld (Cullen, 2020), increasingly urgent.

In addition, older people are more at risk from the effects of COVID-19 because they tend to lack the competences needed to use digital tools effectively; people from remote and rural areas are disadvantaged through lack of access to high speed broadband, and people from poorer backgrounds can't afford the technology - with many families from poorer backgrounds unable to do home education with their children (Cullen, 2020). People with cognitive impairments are particularly vulnerable to these physical, mental and social effects of the pandemic. Their cognitive impairment can limit their

understanding of rapidly disseminated information which is intended to protect them resulting in a higher dependancy on other people and increased vulnerability to exploitation by others in situations where the usual community supports no longer function to protect them (Courtenay and Perera, 2020). This is particularly true when using on-line commercial services as it can be challenging for people with cognitive impairment to process complex information can struggle with poorly-designed and wordy web pages.

More broadly, even though they show interest in using digital tools and stand to gain a lot from using them, people with cognitive disabilities are half as likely to own a computer and one quarter as likely to use the Internet than the population at large (Friedman \& Bryen, 2007) creating an additional barrier to inclusive web accessibility and an absence of cognitive impaired, user-centred design (Schulz et. al., 2015). Indeed, there is an increasing body of evidence to show that technology designers and developers have little or no knowledge of the challenges people with cognitive disabilities face when using technologies (Disability Rights Commission, 2004). Another related issue is that people with cognitive impairments are frequently bundled together as a homogenous group, so that designers and developers impose a 'one size fits all' solution not only on a diverse spectrum of people whose digital challenges and needs stem from varied and often complex origins but on people who present with a wide spectrum and intensity - of impairment, from very mild to very severe (Neven, 2010; Collings et. al., 2018).

\section{Web Accessibility for People with Cognitive Impairment}

A crucial first step is to develop a baseline understanding of the current state of web accessibility for people with cognitive disabilities. Thus, the purpose of this review is to conduct baseline research to understand the factors and processes that inhibit access to online content and services for people with 
cognitive impairments. The labels cognitive disability and cognitive impairment are used to describe a wide range of issues associated with mental processes. People with cognitive disabilities experience difficulties with memory, executive function, reasoning, attention, language, literacy, perception, emotional expression, and comprehension for a variety of reasons including, but not limited to, intellectual disabilities, brain injuries, dementia, and other age-related issues (Braddock, et.al., 2004; W3C, 2021).

The World Wide Web Consortium (W3C), an international community committed to developing open standards for web development, launched the Web Accessibility Initiative (WAI) to improve web accessibility for people with disabilities. Through WAI, W3C developed the Web Content Accessibility Guidelines (WCAG). W3C's Cognitive and Learning Disability (COGA) Task Force have identified which of the current guidelines support users with cognitive disabilities. Additionally, the COGA Task Force has published recommended scenarios for investigation in user group research around eight specific cognitive disabilities: Dyslexia, Aphasia, Non-Verbal, Down Syndrome, Autism, Dyscalculia, Aging-Related Cognitive Decline, Attention Deficit Disorder (ADD/ ADHD). These recommendations shape our definition of web accessibility for people with cognitive disabilities - that web users with cognitive disabilities can navigate and engage with websites, web tools, and web technologies to accomplish tasks associated with health, education, and employment, to communicate, to use the web of things and web systems, and to access critical information.

In light of the above this time limited review will act as an interventions audit by collecting, collating and analysing examples of good practices - 'interventions'- including examples of platforms and tools - that have the potential to support inclusive web accessibility. The goal of this action is to find out what strategies, platforms and tools work for which people, in what circumstances, and how. The following review question will guide this work: What is the state-of-the-art of interventions that support web accessibility for citizens, ages 9 and up, living with cognitive impairment?

In order to answer this overacting review question the following secondary review questions will be attended to:

- What are examples of good practices - 'interventions'- including examples of platforms and tools that have the potential to support inclusive web accessibility for people with cognitive disabilities?

- What is the impact of successful initiatives, interventions or programmes on supporting inclusive web accessibility for people with cognitive impairment?

- What are the similarities and differences between successful initiatives, interventions or programmes supporting web accessibility for people with cognitive impairment in the different sectors (i.e. education, health, commerce, employment, etc...)?

- What are the drivers and barriers, or supports and constraints, for web accessibility for people with cognitive impairment?

- What are the key characteristics of successful initiatives, interventions or programmes on supporting inclusive web accessibility for the population under this study? 
- What recommendations can be made for the future initiatives that wish to support web accessibility for citizens living with cognitive impairment?

\section{Methods}

\section{Protocol and Registration}

This rapid evidence assessment is informed by publications on best practice for such work from the World Health Organisation (WHO) and UK Department for Environment, Food \& Rural Affairs (DEFRA). This protocol has been submitted to the International Prospective Register for Systematic Reviews PROSPERO and is pending formal registration. At present, there is no universally recognised reporting process for rapid reviews and consequently this protocol has adapted the PRISMA-P and associated checklist (Additional File 2) as a framework to report on the findings of the review that typically underpin systematic reviews.

\section{Inclusion \& Exclusion Criteria}

The research team are interested in identifying, synthesising and considering evidence of the impact of initiatives, interventions or programmes that have the potential to support inclusive web accessibility. The research team is primarily interested in the impact of initiatives, interventions or programmes in four key areas: accessing emergency resources; life skill development; self-development through education; and health services and related support.

The research team will consider studies that are qualitative, quantitative or mixed methods. Studies that do not include imperial data will be considered for inclusion in this review as extant programmes that are not be imperial in nature may offer useful insights into best practice or experience particular to individual cognitive impairments or combinations of cognitive impairments that individuals experience. The project team will consider with such studies for inclusion on a case-by-case basis. Following on from this the following inclusion and exclusion criteria have been structured using the PICO (Population, Intervention, Comparison and Outcome) model. These criteria are set out here:

\section{Population}

The review will focus on citizens ages 9 and up who live with cognitive impairment. The lower age limit of 9 years was set to capture the year prior to the transition from primary school to secondary school across the multiple jurisdictions represented in this research partnership. No upper age limit was set, because the need for web accessibility when living with cognitive impairment does not diminish or stop at any particular age. In the instance where studies include participants that are determined to be outside of the study age range, if the mean age range of the study is 9 years of age or greater, the study will be included subject to agreement by the project team members. Studies that are returned that do not focus exclusively on citizens with cognitive impairments and where the datasets presented by the study are not presented separately, such studies will be excluded from this review. 


\section{Intervention}

For the purpose of this review, interventions will only be considered if they adhere to the target population parameters set out in the previous section. Studies that report on interventions implemented within the lifeworld experiences of citizens who experience cognitive impairment such as those set out by WC3. Studies that incorporate combinations of the above will also be included.

\section{Comparison}

While it is unlikely that studies with concurrent control or comparison groups will emerge in the review process, if they do present then they will be included. If sub-group analysis is possible this will be explored in relation to the specific impact of initiatives, interventions and programmes on citizens who experience cognitive impairment.

\section{Outcomes}

The impact of initiatives, interventions and programmes on citizens who experience cognitive impairment. will be included as will studies that report on the impact of initiatives, interventions and programmes on the holistic wellbeing of the target population.

\section{Search Strategy}

At the outset of this study the research team carried out a search for existing systematic reviews and meta-analysis in this area. This initial search revealed that no reviews or meta-analysis focusing on initiatives, interventions or programmes that support web accessibility for people with cognitive impairment exists. This systematic, rapid evidence assessment, review will employ a search strategy using defined terms within agreed search strings (Additional File 1). Databases deemed most likely by the research team to return a high number of relevant results will be searched. It is acknowledged by the research team that additional searches may be warranted. Initially, the following databases will be searched: Web of Science, SCOPUS, EBSCOhost, ERIC and ProQuest.

Internet searching through Google Scholar will be carried out as well as forward and backward tracking of citations from studies that are included in the review. Hand searches of journals will be carried out if warranted. Where necessary additional follow up contact with authors, experts and research groups will further inform this review. The review will only include peer reviewed studies published in English, however, if a study published in an alternate language is deemed relevant it will be included. The results of the search will be downloaded and saved to Mendeley and duplicates will be removed. The titles and abstracts of all remaining studies will be screened by two independent reviewers informed by the study inclusion and exclusion criteria. Where a decision to include or exclude cannot be agreed an additional reviewer will adjudicate. All results, screening process results will be documented in tabular form and communicated in a PRISMA flow diagram. 
In addition, the research team considered it necessary to carry out a grey literature search due to the nature of the work being investigated. The expertise within the research team indicated that many programmes that support people with cognitive impairments do not formally report their work through academic dissemination pathways. Consequently it is intended that a grey literature search will be carried out to supplement that findings of study. In contrast to the focus on studies published in English for peer review returns, the grey literature search will actively seek out returns across all languages of the European Union.

\section{Data Extraction and Quality Appraisal}

After the final research returns have been collated two independent reviewers will read all titles and abstracts after duplicates have been removed. The titles and abstracts will be screened in accordance with inclusion and exclusion criteria. Where it is not possible to determine relevance from the title and abstract alone, at this stage the full paper will be accessed to determine whether it should be included. Where there is disagreement between reviewers a third independent reviewer will review the paper and attempts will be made to resolve the disagreement through discussion. If at this point there is still disagreement the paper will be retained for future reference.

The next step in this process will be full paper screening. Where a paper is deemed not to meet the review inclusion criteria it will be rejected following the dual appraisal method as described above. Papers that meet the inclusion criteria will have the following data extracted and highlighted in table format with brief descriptions where appropriate:

- Authorship

- Year of publication will be listed. This will be a time limited study of 10 years.

- Aims of the study

- Sampling strategy and characteristics (also process of recruitment, drop out)

- Methodology

- Description of the interventions

- Key findings/outcomes

- Overall quality rating of the study using the CASP Appraisal Tool Checklist (2018).

\section{Data Analysis and Synthesis}

It is anticipated by the research team that a wide range of study designs and outcomes will be returned as part of this review. The data extracted from this review will be presented in a thematic narrative format. It is hoped that this will include graphics and/or evidence tables. Where the review team determines that the best course of action is to use statistical summaries this approach will be implemented however, it is the view of the review team, informed by domain expertise, that this is unlikely. 


\section{Assessing the Risk of Bias}

Initially, each study will be reviewed by two independent reviewers for quality using the CASP Appraisal Tool Checklist (2018). The intention of the review is not to exclude studies on the basis of bias rather it is to note the quality of the studies being reviewed. To examine for selective reporting of results, papers will be examined for indicators of multiple use of samples included in the papers. For example, this may include indicators that data has been drawn from larger projects, a published thesis or other papers. Papers that are determined to fall under this category will be checked against alternate sources, where available, to outcomes relevant to this review are being presented. Finally, GRADE-CERQual guidelines will also be followed in the assessment of outcome quality for methodological limitations, coherence, adequacy of data and relevance.

\section{Amendments to the Protocol}

Where there are unavoidable significant deviations from this protocol, such amendments will be recorded and reported within the result of the review.

\section{Discussion}

This rapid review protocol will focus on citizens ages 9 and up who live with cognitive impairment and establish a baseline for best practice in supporting web accessibility for people with cognitive impairments. It will achieve this by providing a time limited state-of-the-art evidence report, specific to the challenges people with cognitive impairment, that will help those involved in policy development, policy response initiatives and localised activity. It is intended that, depending on the outcome of the review process, additional opportunities for innovation and/or research may be communicated to relevant stakeholders and policy makers.

\section{Abbreviations}

WAl: Web Accessibility Initiative

W3C: World Wide Web Consortium

WCAG: Web Content Accessibility Guidelines

COGA: Cognitive and Learning Disability

ADD: Attention Deficit Disorder

ADHD: Attention Deficit Hyperactivity Disorder

PICO: Population, Intervention, Comparison and Outcome

PRISMA-P: Preferred Reporting Items for Systematic Reviews and Meta Analyses 
REA: Rapid Evidence Assessment

\section{Declarations}

\section{Ethics approval and consent to participate}

Not applicable

\section{Consent for publication}

Not applicable

\section{Availability of data and material}

Not applicable

\section{Competing interests}

None

\section{Funding}

This work was co-funded by European Union through the PPPA-COGN-2020 programme. Agreement Number: LC-01624438/101018048

\section{Authors' contributions}

$\mathrm{PF}$, is the principal investigator of this review and contributed to review conception, refinement of the design and writing the manuscript. SG, JC, MC, JS, GH, CC, EH \& AH contributed to review conception, refinement of the design and writing the manuscript. PF, SG \& JC collaboratively lead the drafting the manuscript with regular review and engagement with all authors. All authors were involved in the conception, design and iterative refinement of the final manuscript.

\section{Acknowledgements}

The authors would like to acknowledge the support of their individual affiliate institutions regarding the development of this research project.

\section{References}

1. Barnard, S., \& Beyer, S. (2009). Barriers to using personalised technology with people with learning disabilities. Journal of Assistive Technologies

2. Borg, J., Lantz, A., \& Gulliksen, J. (2015). Accessibility to electronic communication for people with cognitive disabilities: a systematic search and review of empirical evidence. Universal Access in the Information Society, 14(4), 547-562 
3. Braddock, D., Rizzolo, M. C., Thompson, M., \& Bell, R. (2004). Emerging technologies and cognitive disability. Journal of Special Education Technology, 19(4), 49-56.

4. Collings, S., Dew, A., \& Dowse, L. (2018). Unpacking the complexity of planning with persons with cognitive disability and complex support needs. Journal of Applied Research in Intellectual Disabilities, 31(1), 142-151.

5. Courtenay, K., \& Perera, B. (2020). COVID-19 and people with intellectual disability: impacts of a pandemic. Irish Journal of Psychological Medicine, 37(3), 231-236.

6. Cullen, J (2020). Solutions for digital inclusion - the MEDICI project. Eutopia Conference, July 20202.

7. Disability Rights Commission. (2004). The web: Access and inclusion for disabled people; a formal investigation. The Stationery Office.

8. Flynn, P., McHugh, M., McHugh, S., Flanagan, E., \& Hall, T. (2016) Leading Through Design:

Educational Design Research for Authentic and Meaningful Impact in Naturalistic Contexts. European Conference on Educational Research 2016. Dublin, Ireland.

9. Friedman, M. G., \& Bryen, D. N. (2007). Web accessibility design recommendations for people with cognitive disabilities. Technology and disability, 19(4), 205-212

10. Neven, L. (2010). 'But obviously not for me': robots, laboratories and the defiant identity of elder test users. Sociology of health \& illness, 32(2), 335-347.

11. Schulz, R., Wahl, H. W., Matthews, J. T., De Vito Dabbs, A., Beach, S. R., \& Czaja, S. J. (2015).

Advancing the aging and technology agenda in gerontology. The Gerontologist, 55(5), 724-734

12. W3C (2021) How People with Disabilities Use the Web. https://www.w3.org/WAl/people-use-web/

13. World Economic Forum. (2020). The Future of Jobs Report 2020. Geneva: World Economic Forum.

\section{Supplementary Files}

This is a list of supplementary files associated with this preprint. Click to download.

- LIVEITSYSTEMATICREVIEWJOUNRALAddtionalFile1.docx

- LIVEITSYSTEMATICREVIEWPRISMAP2015checklist.docx 